\title{
Publisher Correction: Personalized early detection and prevention of breast cancer: ENVISION consensus statement
}

Nora Pashayan (D), Antonis C. Antoniou, Urska Ivanus (D), Laura J. Esserman, Douglas F. Easton (D), David French (D), Gaby Sroczynski, Per Hall, Jack Cuzick, D. Gareth Evans, Jacques Simard (D, Montserrat Garcia-Closas, Rita Schmutzler, Odette Wegwarth, Paul Pharoah (D), Sowmiya Moorthie, Sandrine De Montgolfier (D), Camille Baron, Zdenko Herceg (D, Clare Turnbull, Corinne Balleyguier, Paolo Giorgi Rossi, Jelle Wesseling, David Ritchie (iD, Marc Tischkowitz, Mireille Broeders, Dan Reisel, Andres Metspalu (D), Thomas Callender (D), Harry de Koning (D), Peter Devilee, Suzette Delaloge (D), Marjanka K. Schmidt (D) and Martin Widschwendter (D)

Nature Reviews Clinical Oncology (2020) https://doi.org/10.1038/s41571-020-0388-9 Published online 18 June 2020

This paper was originally published without open access. As of the date of this correction, the paper is available online as an open-access paper under a Creative Commons Attribution 4.0 International License.

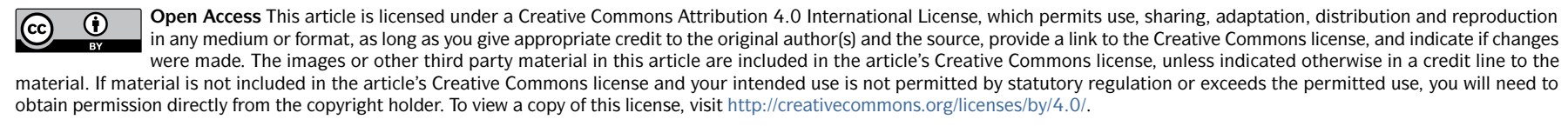

https://doi.org/10.1038/s41571-020-0412-0 I Published online 29 June 2020

(c) The Author(s) 2020 